This is the post peer-review accepted manuscript of:

A. Guerra, D. Dardari and P. M. Djurić, "Joint Indoor Localization and Navigation of UAVs for Network Formation Control," 2018 52nd Asilomar Conference on Signals, Systems, and Computers, Pacific Grove, CA, USA, 2018, pp. 13-19.

doi: https://doi.org/10.1109/ACSSC.2018.8645291

The published version is available online at:

http://ieeexplore.ieee.org/stamp/stamp.jsp?tp=\&arnumber=8645291\&isnumber $=8645065$

(C) 2018 IEEE. Personal use of this material is permitted. Permission from IEEE must be obtained for all other uses, in any current or future media, including reprinting/republishing this material for advertising or promotional purposes, creating new collective works, for resale or redistribution to servers or lists, or reuse of any copyrighted component of this work in other works 


\title{
Joint Indoor Localization and Navigation of UAVs for Network Formation Control
}

\author{
Anna Guerra*, Davide Dardari*, and Petar M. Djurić ${ }^{\dagger}$ \\ *DEI-CNIT, University of Bologna, Via dell’Universitá, 47522 Cesena, Italy. E-mail: \{anna.guerra3, davide.dardari\}@unibo.it \\ $\dagger$ ECE, Stony Brook University, Stony Brook, NY 11794, USA. E-mail: petar.djuric@stonybrook.edu
}

\begin{abstract}
In this paper, we propose a joint indoor localization and navigation algorithm to enable a swarm of unmanned aerial vehicles (UAVs) to deploy in a specific spatial formation in indoor environments. In the envisioned scenario, we consider a static user acting as a central unit whose main task is to acquire all the UAV measurements carrying position-dependent information and to estimate the UAV positions when there is no existing infrastructure for positioning. Subsequently, the user exploits the estimated positions as inputs for the navigation control with the aim of deploying the UAVs in a desired formation in space (formation shaping). The user plans the trajectory of each UAV in real time, guaranteeing a safe navigation in the presence of obstacles. The proposed algorithm guides the UAVs to their desired final locations with good accuracy.
\end{abstract}

Index Terms-Anchor-free localization, UAV network, formation shaping control.

\section{INTRODUCTION}

In the last couple of decades, multi-agent networks have often been studied especially in the robotic and control research fields [1], [2]. Initially, the interest was generated by military applications because autonomous agents, able to operate without a pilot, represent a valid alternative to human soldiers in high-risk missions [3]. In this context, trajectory optimization and motion control have been analyzed for unmanned aerial vehicles (UAVs), unmanned ground vehicles (UGVs), and autonomous underwater vehicles (AUVs), together with their capability of formation shaping and maintenance [4], [5]. Another broad domain of applications has been in space exploration, where the autonomy and flexibility of the agents play crucial roles [6].

Recently, the idea of using swarms or fleets of autonomous vehicles (AVs) with hundreds of agents has been put forth because of the necessity of enhancing the robustness in completing assigned tasks to AVs. Further, such fleets permit to allocate different tasks to different sub-swarms: for example, the encirclement of an obstacle by a group of AVs and the arrival at the base by another [7]. This work has opened research on intelligent agents for outdoor civil applications, such as delivery, logistic, precision agriculture, emergency or post-disaster events [8]-[10].

In our previous work [11], the UAVs fly outdoors and their mission is to navigate in a way that maximizes the capability of localizing a target. Going a step forward, one could easily imagine a setting where the UAVs enter a building with harsh propagation conditions, with many obstacles and a drastically
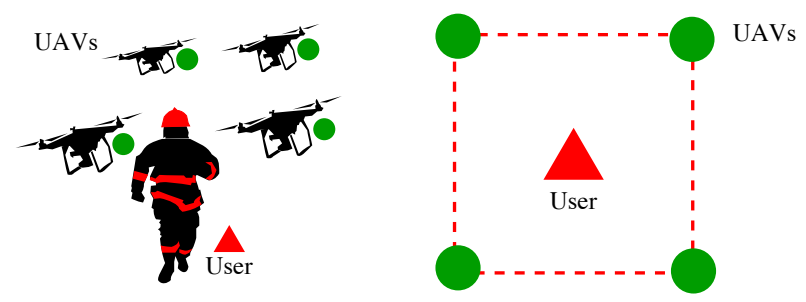

Fig. 1: An indoor application scenario using a user-centric network of UAVs.

reduced space for maneuvering. Indoor environments provide many challenges from scientific and engineering points of view because the UAVs do not have access for localization by a global reference system, such as by satellites (GPS) or by an ad-hoc positioning infrastructure (anchor nodes). Therefore, in addition to their traditional navigation tasks, they must perform anchor-free localization based on inter-UAV relative measurements [12]. This, in turn, will require superior sensing and communication capabilities than those needed in outdoor applications.

In an anchor-free scenario, the UAVs have to measure some inter-UAV position-dependent quantities, such as distances and angles, and then, communicate them to other UAVs or to a central node (if it exists). The latter will be responsible for processing the received data and for inferring the positions of all the UAVs of the network. In this regard, several anchor-free localization algorithms have been proposed in the literature, as, for example, the ones described in [13] and the references therein.

While addressing the problems of localization and navigation, physical and dynamical constraints must be taken into account. The UAVs are expected to be lightweight, the hardware on board cannot be complex (low complexity), and the necessary computations have to be fast. For example, the interval between the instant in which the current position is estimated and the next position is reached should be kept as short as possible (low latency). Obviously, satisfying these requirements may come at the expense of a lower localization and formation accuracy, and, thus, it is important to analyze the trade-off between the technological issues and the attainable performance.

In this paper, we study a network of multiple UAVs and 
a central user that can either be a drone, a mobile device, or a terrestrial vehicle, operating in indoor environments (see Fig. 1). The goal of the network is twofold: first, the UAVs should be localized with respect to the user (infrastructure-less localization) and, subsequently, they should deploy according to a desired topology (formation shape) and guidance by the user. The system is centralized: all the computational load is handled by the user, and in this way the UAVs can save their energy and complete parallel tasks while waiting for the navigation commands. Further, we suppose that all the UAVs are able of exchanging distance or angle-of-arrival (AOA) measurements with all the other UAVs in the network and also with the user. The accuracy of such measurements clearly depends on the technology available on board. For example, for distance estimation, the ultrawide bandwidth (UWB) technology provides higher performance with respect to what can be obtained by received signal strength (RSS) measurements [14], [15]. Likewise, the availability of antenna array measurements permits to achieve much more precise AOA estimates than those obtained from power observations. In [16]-[18], it is shown how AOA information can be extrapolated from RSS data by exploiting the possibility of pointing the UAV's sensor antenna in different spatial directions using UAV rotations and by searching for the RSS peak. Here, we chose to work with simple sensors and studied the achievable localization and formation accuracy based on their measurements.

The paper is organized as follows. Section II illustrates the problem, Sec. III describes the characterization of the relative localization error, Sec. IV presents the approach used for the control of the UAVs, Sec. V describes the results and, finally, Sec. VI draws conclusions and discusses possible future extensions.

\section{Problem Statement}

In this paper, we aim at studying how to navigate UAVs in forming a network with a desired topology with respect to a static user in indoor environments where GPS signals are not available and in the absence of an ad-hoc positioning infrastructure. A possible application is the deployment of an autonomous team of UAVs acting as radio "flying eyes" and helping a rescuer (user) in completing his/her job, for example, by enhancing his/her capability for sensing the surrounding environment, see Fig. 1.

The network is composed of a single user and $N$ UAVs. The UAVs are equipped with radio sensors and exchange ranging or bearing (AOA) measurements with each other and with the user. The user acts as the center of the network and as a (centralized) processing unit. More specifically, it performs two high-level tasks: the first is the relative localization of all the UAVs that compose the network with respect to itself (namely T1 in Fig. 2); and the second is the computation of control signals for UAV navigation (namely T2 in Fig. 2). To do this, the user collects all the measurements from the UAVs and estimates their relative positions with respect to an arbitrary selected coordinate system. Then, it evaluates the

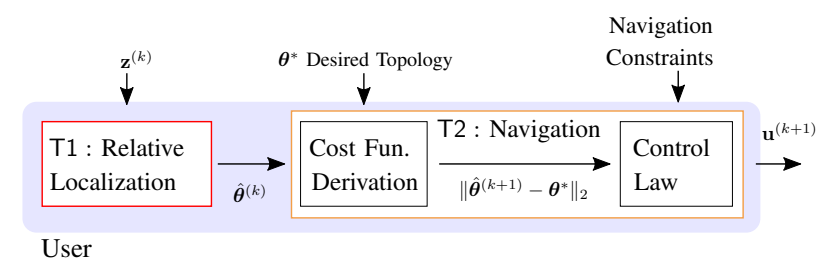

Fig. 2: The user's block-diagram for joint localization and navigation.

distance to be travelled by each UAV to attain the desired formation and sends back the navigation commands to each UAV at each time instant.

To formalize the problem, we indicate with

$$
\boldsymbol{\theta}^{(k)}=\left[\left(\mathbf{x}^{(k)}\right)^{\top},\left(\mathbf{y}^{(k)}\right)^{\top}\right]^{\top}
$$

the actual position of all the UAVs composing the network at time instant $k$ (state vector), where

$$
\begin{aligned}
\mathbf{x}^{(k)} & =\left[x_{1}^{(k)}, \ldots, x_{i}^{(k)}, \ldots, x_{N}^{(k)}\right]^{\top}, \\
\mathbf{y}^{(k)} & =\left[y_{1}^{(k)}, \ldots, y_{i}^{(k)}, \ldots, y_{N}^{(k)}\right]^{\top}
\end{aligned}
$$

are their $x$ and $y$ Cartesian coordinates, respectively. For notation simplicity, we consider a two-dimensional scenario - the extension to a three-dimensional setting is straightforward. We assume that the user is located at the origin of the coordinate system (defined below), i.e., $\mathbf{p}_{0}^{(k)}=$ $\left[x_{0}^{(k)}, y_{0}^{(k)}\right]^{\top}=[0,0]^{\top}$.

The locations of the UAVs with respect to the user are unknown. Based on the exchanged measurements between the UAVs, such positions can be inferred by considering a coordinate system with the $x$-axis defined by a baseline centered at the user position, i.e., $\mathbf{b}^{(k)}=\left[\mathbf{p}_{0}^{(k)}, \hat{\mathbf{p}}_{1}^{(k)}\right]^{\mathrm{T}}$ with $\hat{\mathbf{p}}_{1}^{(k)}=\left[\hat{d}_{1}^{(k)}, 0\right]^{\top}$ being the position of the first UAV. The $y$-axis is obtained by rotating the $x$-axis counterclockwise by an angle of $\pi / 2$.

In the sequel, we denote with $N_{\mathrm{r}}$ and $N_{\mathrm{u}}$ the number of UAVs whose positions are known and unknown, respectively. In our case, $N_{\mathrm{r}}=1$ and $N_{\mathrm{u}}=N-1$. The user estimates the corresponding $2 N_{\mathrm{u}}$ unknown parameters and stacks their values in

$$
\hat{\boldsymbol{\theta}}^{(k)}=\left[\left(\hat{\mathbf{x}}^{(k)}\right)^{\top},\left(\hat{\mathbf{y}}^{(k)}\right)^{\top}\right]^{\top}=\boldsymbol{\theta}^{(k)}+\boldsymbol{\omega}^{(k)},
$$

where $\hat{\mathbf{x}}^{(k)}$ and $\hat{\mathbf{y}}^{(k)}$ are the vectors with the estimated $x$ and $y$ coordinates, and $\boldsymbol{\omega}^{(k)}$ is the position estimation error.

Once the UAV positions are estimated, the user provides the control signals to attain the desired configuration of the UAVs with respect to the user, i.e., $\boldsymbol{\theta}^{*}=\left[\left(\mathbf{x}^{*}\right)^{\top},\left(\mathbf{y}^{*}\right)^{\top}\right]^{\top}$, which does not depend on time. 
Finally, the formation problem can be formulated as a minimization problem, that is,

$$
\begin{aligned}
\left(\boldsymbol{\theta}^{(k+1)}\right)^{\star}=\underset{\hat{\boldsymbol{\theta}}^{(k+1)} \in \mathbb{R}^{2 N}}{\arg \min } & \left\|\hat{\boldsymbol{\theta}}^{(k+1)}-\boldsymbol{\theta}^{*}\right\|, \\
\text { subject to } \quad & d_{i j}^{(k+1)} \geq d_{\mathrm{S}} \\
& \mathcal{T} \cap \mathcal{O}=\varnothing \\
& \left\|\hat{\boldsymbol{\theta}}_{i}^{(k+1)}-\hat{\boldsymbol{\theta}}_{i}^{(k)}\right\|=v
\end{aligned}
$$

for $i=0, \ldots, N, j \neq i$, where $d_{i j}^{(k)}$ is the distance between the $i$ th and $j$ th UAV at time instant $k$ as perceived by the on-board proximity sensors, and $d_{\mathrm{S}}$ is the inter-UAV safety distance. Moreover, $\mathcal{T}$ represents the set of trajectories followed by all the UAVs and $\mathcal{O}=\bigcup_{i=1}^{N_{\text {obs }}} \mathcal{O}_{i}$ the set of all obstacles from which the UAVs should keep a safety distance equal to $d_{O}$. Finally, the last constraint imposes a constant UAV speed equal to $v$. Then, the control signal to be applied at the $i$ th UAV to satisfy (5) is

$$
\mathbf{u}^{(k+1)}=\left(\boldsymbol{\theta}^{(k+1)}\right)^{\star}-\hat{\boldsymbol{\theta}}_{i}^{(k)}
$$

where its $i$ th element $\mathbf{u}_{i}^{(k+1)}=\left[\Delta x_{i}^{(k+1)}, \Delta y_{i}^{(k+1)}\right]^{\top}$ is the spatial step to be taken by the $i$ th UAV to lower the distance toward the final desired position.

\section{INFRASTRUCTURE-LESS LOCALIZATION}

In this section, we characterize the position estimation error in (4). In general, the relative localization uncertainty depends on the implemented estimator. To gain an insight about the best attainable performance, we consider the error having zeromean Gaussian distribution with a covariance matrix $\boldsymbol{\kappa}^{(k)}$ equal to the relative Cramér-Rao lower bound (CRLB) of the UAV positions. In the following we derive the log-likelihood function starting from the specific observation model and, then, we derive the CRLB.

\section{A. Observation model}

We denote with $\mathbf{z}^{(k)}$ the vector containing all the measurements available at the user at time instant $k$,

$$
\mathbf{z}^{(k)}=\left[\ldots, z_{i j}^{(k)}, \ldots\right]^{\top},
$$

with $i, j=0, \ldots, N$ and where we consider the measurement between the $(i, j)$ th pair of UAVs only once to not burden the processing of measurements, i.e., $j>i$. The generic element is the measurement between the $i$ th and $j$ th UAV given by

$z_{i j}^{(k)}=$

$$
\begin{cases}h_{i j}^{(k)}\left(\boldsymbol{\theta}^{(k)}\right)+v_{i j}^{(k)} & \text { ranging } \\ p_{i j}^{(k)}\left(h_{i j}^{(k)}\left(\boldsymbol{\theta}^{(k)}\right)+v_{i j}^{(k)}\right)+\left(1-p_{i j}^{(k)}\right) q_{i j}^{(k)}, & \text { bearing }\end{cases}
$$

where $p_{i j}^{(k)}=\{1,0\}=\{$ LOS, NLOS $\}$ indicates the presence or absence of a non line-of-sight (NLOS) propagation condition between these UAVs, $q_{i j}^{(k)} \sim \mathcal{U}(0,2 \pi)$ models the presence of outliers due to multipath between them, and $h_{i j}^{(k)}\left(\boldsymbol{\theta}^{(k)}\right)$ is a function of the state vector, where

$$
\begin{aligned}
& h_{i j}^{(k)}\left(\boldsymbol{\theta}^{(k)}\right)= \\
& \left\{\begin{array}{l}
d_{i j}^{(k)}=\sqrt{\left(\Delta x_{i j}^{(k)}\right)^{2}+\left(\Delta y_{i j}^{(k)}\right)^{2}}, \\
\phi_{i j}^{(k)}=\arctan \left(\frac{\Delta y_{i j}^{(k)}}{\Delta x_{i j}^{(k)}}\right),
\end{array}\right.
\end{aligned}
$$

ranging,

bearing,

with $d_{i j}^{(k)}$ and $\phi_{i j}^{(k)}$ being the distance and the angle between the $i$ th and $j$ th UAV at time instant $k$, with $\Delta x_{i j}^{(k)}=x_{i}^{(k)}-x_{j}^{(k)}$ and $\Delta y_{i j}^{(k)}=y_{i}^{(k)}-y_{j}^{(k)}$. The measurement error is indicated with $v_{i j}^{(k)}$ modeled as a zero-mean Gaussian random variable with a standard deviation given by

$$
\sigma_{i j}^{(k)}= \begin{cases}\sigma_{0, \mathrm{r}} \cdot d_{i j}^{(k)}\left(\boldsymbol{\theta}^{(k)}\right), & \text { ranging, } \\ \sigma_{0, \mathrm{~b}}, & \text { bearing, }\end{cases}
$$

where the ranging model is distance dependent, with $\sigma_{0, \mathrm{r}}$ being the ranging standard deviation at the reference distance (i.e., $d_{0}=1 \mathrm{~m}$ ) and the bearing model is considered constant with respect to the state vector. For RSS-based ranging observations, it is possible to model the ranging standard deviation as $\sigma_{0, \mathrm{r}}=\frac{\ln 10}{10 \alpha}\left(\sigma_{\mathrm{sh}}+\left(1-p_{i j}^{(k)}\right) \sigma_{\mathrm{b}}\right)$, where $\alpha$ is the path-loss exponent, $\sigma_{\text {sh }}$ corresponds to shadowing in $\mathrm{dB}$ and $\sigma_{\mathrm{b}}$ represents a bias term due to the NLOS condition.

Consequently, we can write the likelihood function as $f\left(\mathbf{z}^{(k)} \mid \boldsymbol{\theta}^{(k)}\right)=\mathcal{N}\left(\mathbf{z}^{(k)} ; \mathbf{h}^{(k)}, \mathbf{Q}^{(k)}\right)$ where $\mathbf{h}^{(k)}=$ $\left[\ldots, h_{i j}^{(k)}, \ldots\right]^{\top}$ contains the expected ranging and bearing values and $\mathbf{Q}^{(k)}=\operatorname{diag}\left(\ldots,\left(\sigma_{i j}^{(k)}\right)^{2}, \ldots\right)$ is the diagonal covariance matrix. Given this observation model, the loglikelihood function available at the user at the $k$ th time slot is

$$
\Lambda\left(\mathbf{z}^{(k)} \mid \boldsymbol{\theta}^{(k)}\right)=\sum_{i=0}^{N} \sum_{\substack{j=0 \\ j>i}}^{N} \ln f\left(z_{i j}^{(k)} \mid \boldsymbol{\theta}^{(k)}\right) .
$$

In the literature, different localization algorithms have been proposed for dealing with the absence of an ad-hoc positioning infrastructure. To name a few, an anchor-free localization using hop count (AFL) has been proposed in [19], maximum likelihood and least square schemes are described in [20], and Bayesian schemes are investigated in [13]. Surveys on infrastructure-less positioning can be found in [12], [13].

In the rest of the paper, we consider an unbiased estimator with a covariance matrix defined by the relative CRLB, derived in the following section. More specifically, we model the position estimates as in (4) with $\boldsymbol{\omega}^{(k)} \sim \mathcal{N}\left(\mathbf{0}, \boldsymbol{\kappa}^{(k)}\right)$, and $\boldsymbol{\kappa}^{(k)}=\boldsymbol{\kappa}\left(\hat{\boldsymbol{\theta}}^{(k-1)}\right)$ being the corresponding CRLB evaluated according to the previous position estimates. This choice allows for investigation of the formation performance in a more general way without dealing with a specific localization algorithm, and for obtaining the maximum achievable accuracy. 


\section{B. Anchor-free CRLB}

In this section, we evaluate the anchor-free CRLB that will be used to model the covariance matrix of the position estimates in (4). The performance of any unbiased estimator $\hat{\boldsymbol{\theta}}^{(k)}$ can be bounded by the CRLB, namely $\boldsymbol{\kappa}\left(\boldsymbol{\theta}^{(k)}\right)$, defined as [21]

$$
\mathbb{E}\left\{\left[\hat{\boldsymbol{\theta}}^{(k)}-\boldsymbol{\theta}^{(k)}\right]\left[\hat{\boldsymbol{\theta}}^{(k)}-\boldsymbol{\theta}^{(k)}\right]^{\top}\right\} \succeq \mathbf{J}^{-1}\left(\boldsymbol{\theta}^{(k)}\right)=\boldsymbol{\kappa}\left(\boldsymbol{\theta}^{(k)}\right),
$$

where $\mathbf{A} \succeq \mathbf{B}$ means that $\mathbf{A}-\mathbf{B}$ is positive semi-definite, and $\mathbf{J}\left(\boldsymbol{\theta}^{(k)}\right)$ is the $2 N_{\mathrm{u}} \times 2 N_{\mathrm{u}}$ Fisher Information Matrix (FIM), i.e.,

$$
\begin{aligned}
\mathbf{J}\left(\boldsymbol{\theta}^{(k)}\right) & =\mathbb{E}_{\mathbf{z}^{(k)}}\left\{\left[\nabla_{\boldsymbol{\theta}^{(k)}} \Lambda\left(\mathbf{z}^{(k)} \mid \boldsymbol{\theta}^{(k)}\right)\right]\left[\nabla_{\boldsymbol{\theta}^{(k)}} \Lambda\left(\mathbf{z}^{(k)} \mid \boldsymbol{\theta}^{(k)}\right)\right]^{\top}\right\} \\
& =\left[\begin{array}{cc}
\mathbf{J}_{\times \mathrm{x}}^{(k)} & \mathbf{J}_{\times \mathrm{y}}^{(k)} \\
\left(\mathbf{J}_{\times \mathrm{y}}^{(k)}\right)^{\top} & \mathbf{J}_{\mathrm{yy}}^{(k)}
\end{array}\right],
\end{aligned}
$$

with the subscripts $x$ and $y$ indicating the Cartesian position coordinates of the UAVs and where the generic elements of the sub-FIMs are [12]

$$
\begin{aligned}
& {\left[\mathbf{J}_{\times x}^{(k)}\right]_{m n}= \begin{cases}\sum_{i} A_{m i}^{(k)}\left(\Delta x_{m i}^{(k)}\right)^{2} /\left(d_{m i}^{(k)}\right)^{s}, & m=n, \\
-A_{m n}^{(k)}\left(\Delta x_{m n}^{(k)}\right)^{2} /\left(d_{m n}^{(k)}\right)^{s}, & m \neq n,\end{cases} } \\
& {\left[\mathbf{J}_{\mathrm{xy}}^{(k)}\right]_{m n}= \begin{cases}\sum_{i} A_{m i}^{(k)} \Delta x_{m i}^{(k)} \Delta y_{m i}^{(k)} /\left(d_{m i}^{(k)}\right)^{s}, & m=n, \\
-A_{m n}^{(k)} \Delta x_{m n}^{(k)} \Delta y_{m n}^{(k)} /\left(d_{m n}^{(k)}\right)^{s}, & m \neq n,\end{cases} } \\
& {\left[\mathbf{J}_{\mathrm{yy}}^{(k)}\right]_{m n}= \begin{cases}\sum_{i} A_{m i}^{(k)}\left(\Delta y_{m i}^{(k)}\right)^{2} /\left(d_{m i}^{(k)}\right)^{s}, & m=n, \\
-A_{m n}^{(k)}\left(\Delta y_{m n}^{(k)}\right)^{2} /\left(d_{m n}^{(k)}\right)^{s}, & m \neq n,\end{cases} }
\end{aligned}
$$

where $i=0, \ldots, N$ and $m=n=1, \ldots, N_{\mathrm{u}}$. The exponent $s$ is equal to 2 in the case of ranging measurements, and $s=4$ in the case of bearing. The coefficient $A_{m i}^{(k)}$ is defined by

$$
A_{m i}^{(k)}= \begin{cases}\left(1+2 \xi \sigma_{0, \mathrm{r}}\right) /\left(\sigma_{m i}^{(k)}\right)^{2}, & \text { ranging, } \\ 1 /\left(\sigma_{0, \mathrm{~b}}^{2}\right), & \text { bearing }\end{cases}
$$

where $\xi=0$ when there is a model mismatch, i.e., when the dependence of $\sigma_{m i}^{(k)}$ from the state vector $\boldsymbol{\theta}^{(k)}$ in (10) is not considered in the FIM evaluation; otherwise $\xi=1$.

\section{The CONTROL LAW}

The constrained minimization problem in (5) can be solved using the projection gradient method [22]. Then the control signal of the $i$ th UAV is given by

$$
\mathbf{u}_{i}^{(k+1)}=-\gamma \mathbf{P} \nabla_{\hat{\boldsymbol{\theta}}_{i}^{(k)}}\left(\left\|\hat{\boldsymbol{\theta}}_{i}^{(k)}-\boldsymbol{\theta}_{i}^{*}\right\|\right)-\mathbf{N}\left(\mathbf{N}^{\top} \mathbf{N}\right)^{-1} \mathbf{g},
$$

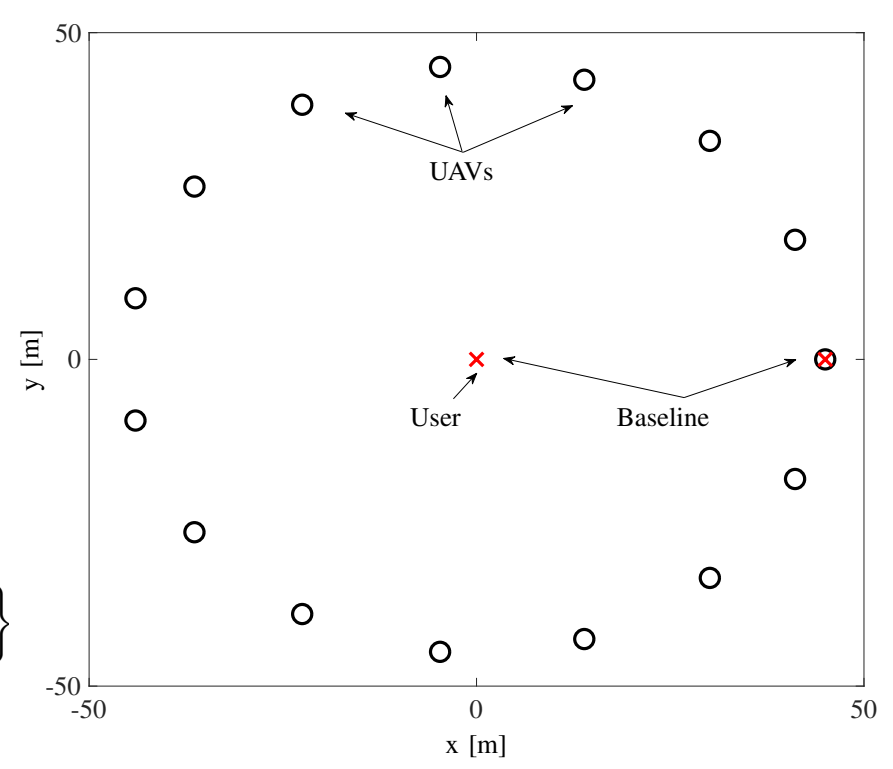

Fig. 3: Infrastructure-less localization scenario.

where $\gamma$ represents the spatial step, and $\nabla_{\hat{\boldsymbol{\theta}}_{i}^{(k)}}(\cdot)$ is the gradient operator with respect to the UAV's estimated position, and it is

$$
\begin{aligned}
& \nabla_{\hat{x}_{i}^{(k)}}\left(\left\|\hat{\boldsymbol{\theta}}_{i}^{(k)}-\boldsymbol{\theta}_{i}^{*}\right\|\right)=\cos \left(\hat{\alpha}_{i}^{(k)}\right), \\
& \nabla_{\hat{y}_{i}^{(k)}}\left(\left\|\hat{\boldsymbol{\theta}}_{i}^{(k)}-\boldsymbol{\theta}_{i}^{*}\right\|\right)=\sin \left(\hat{\alpha}_{i}^{(k)}\right),
\end{aligned}
$$

where $\hat{\alpha}_{i}^{(k)}$ is the angle between the estimated position of the $i$ th UAV and its desired position at time instant $k$. The projection matrix is denoted with $\mathbf{P}=\mathbf{I}-\mathbf{N}\left(\mathbf{N}^{\top} \mathbf{N}\right)^{-1} \mathbf{N}^{\top}$, where $\mathbf{I}$ is the identity matrix and $\mathbf{N}=\nabla_{\hat{\boldsymbol{\theta}}_{i}^{(k)}}(\mathbf{g})$ is the gradient of the activated constraints acquired in $\mathbf{g}=\left[\mathbf{g}_{\mathrm{S}}, \mathbf{g}_{\mathrm{O}}\right]$, where

$$
\begin{aligned}
& \mathbf{g}_{\mathrm{S}}=\mathbf{d}_{\mathrm{S}}-d_{\mathrm{S}}, \quad \mathbf{d}_{\mathrm{S}}=\left\{d_{i j}^{(k)}: d_{i j}^{(k)}<d_{\mathrm{S}}\right\}, \\
& \mathbf{g}_{\mathrm{O}}=\mathbf{d}_{\mathrm{O}}-d_{\mathrm{O}}, \quad \mathbf{d}_{\mathrm{O}}=\left\{l_{i, \mathrm{o}_{j}}^{(k)}: l_{i, \mathrm{o}_{j}}^{(k)}<d_{\mathrm{O}}\right\}
\end{aligned}
$$

with $l_{i, \mathrm{o}_{j}}^{(k)}$ being the minimum distance of the $i$ th UAV from the $j$ th obstacle, indicated as $o_{j}$.

\section{Case Study}

\section{A. Infrastructure-less localization results}

In this section, we report some results on the relative localization performance. We consider that the network of UAVs is distributed in a circumference centered at the user location, i.e., $[0,0]^{\top}$, and with a radius of 45 meters. The $x$ axis was defined by the location of the user and the actual position of the first UAV which is $[45,0]^{\top}$. The positions of these two UAVs are considered known and they are indicated with crosses in Figs. 3-4. All the other UAVs are in unknown positions and they are represented by circles. The measurement standard deviations are set to $\sigma_{0, r}=0.39$ in logarithmic scale 


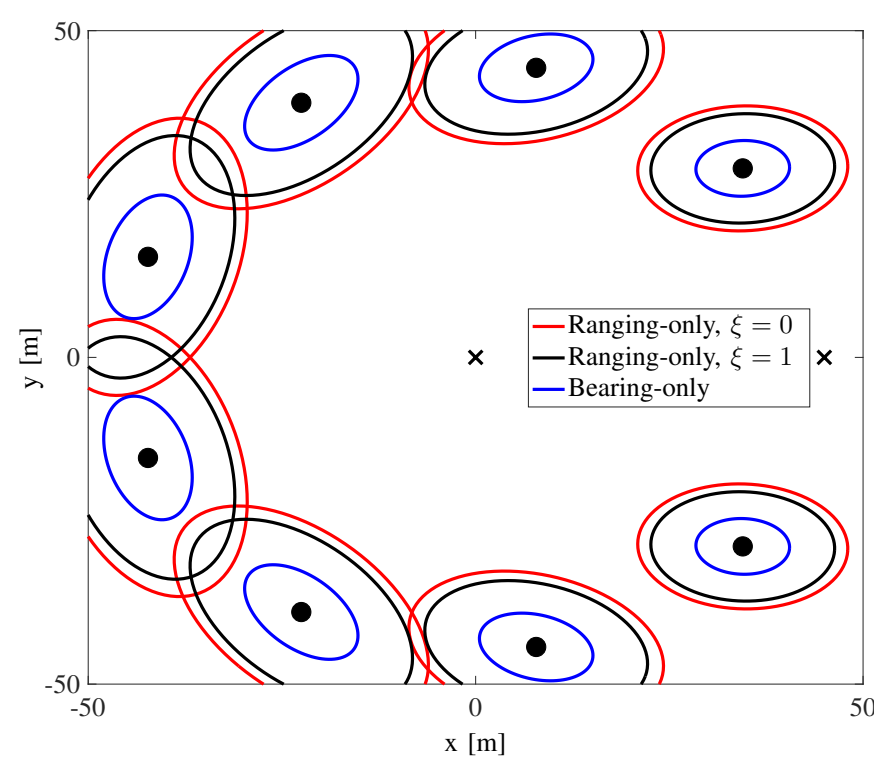

Fig. 4: Localization error ellipses for different measurement models.

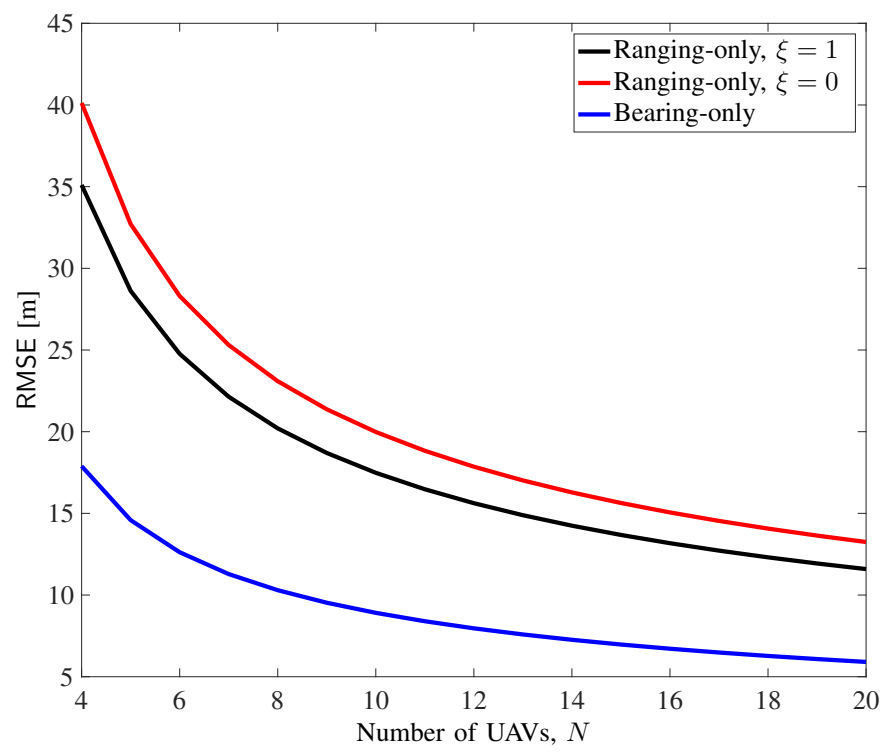

Fig. 5: RMSE as a function of the number of UAVs.

(or, equivalently, $\sigma_{\mathrm{sh}} / \alpha=1.7$ with $\sigma_{\mathrm{sh}}=3.4 \mathrm{~dB}$ ) [12] and $\sigma_{0, \mathrm{~b}}=10^{\circ}[16]-[18]$.

We first calculated the CRLB matrix $\kappa$ by inverting (13) whose sub-FIM elements are expressed in (14). Then we computed the ellipses of the localization uncertainty starting from the CRLB matrix associated to each UAV. For example, for the $i$ th $\mathrm{UAV}$, we have

$$
\boldsymbol{\kappa}_{i}=\left[\begin{array}{ll}
{\left[\boldsymbol{\kappa}_{\mathrm{xx}}(\boldsymbol{\theta})\right]_{i i}} & {\left[\boldsymbol{\kappa}_{\mathrm{xy}}(\boldsymbol{\theta})\right]_{i i}} \\
{\left[\boldsymbol{\kappa}_{\mathrm{yx}}(\boldsymbol{\theta})\right]_{i i}} & {\left[\boldsymbol{\kappa}_{\mathrm{yy}}(\boldsymbol{\theta})\right]_{i i}}
\end{array}\right] .
$$

The directions of the major axes of the ellipses are the eigenvectors of the matrix in (21), whereas their lengths
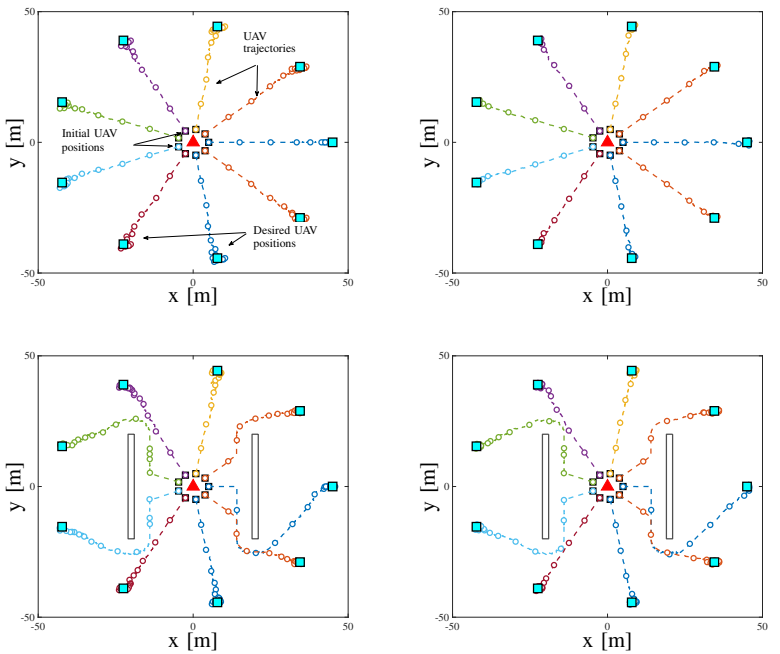

Fig. 6: Example of navigation simulation. Top: LOS propagation scenario; Bottom: NLOS propagation scenario; Left: Ranging-only measurements; Right: Bearing-only measurements.

correspond to their eigenvalues.

Figure 4 presents the results for the following three cases: the red curves refer to ranging observations and ignorance of how the ranging model depends on the state vector, i.e., $\xi=0$; the black curves refer to ranging observations with a perfect knowledge of the observation model; i.e. $\xi=1$; and the blue curves are for the bearing case. As expected, in the case of model awareness, the achievable localization results are better in comparison to the case where the position dependence in the ranging variance is not taken into account by the CRLB computation.

Figure 5 displays the results in terms of RMSE as a function of the number of UAVs. The RMSE was obtained according to

$$
\text { RMSE }=\sqrt{\frac{\operatorname{tr}\left(\boldsymbol{\kappa}_{\mathrm{xx}}(\boldsymbol{\theta})+\boldsymbol{\kappa}_{\mathrm{yy}}(\boldsymbol{\theta})\right)}{N_{\mathrm{u}}}},
$$

where $\operatorname{tr}(\cdot)$ is the trace operator. It is not surprising that by increasing the number of UAVs, due to acquisition of more information, we obtain more accurate position estimates of the UAVs.

The localization results discussed in this section were used as input for processing the navigation commands. In the following, we show the achievable accuracy in network formation when the relative CRLB is used as the variance of the UAV position estimates as indicator of the best performance achievable by any practical estimator.

\section{B. Network formation results}

In this section, we report the performance of the network formation task. More specifically, we suppose that at each time instant $k$, the positions of the UAVs are estimated and the variance is fixed to the CRLB value at that time instant. Then, the control signals are computed as the gradient of the distance 


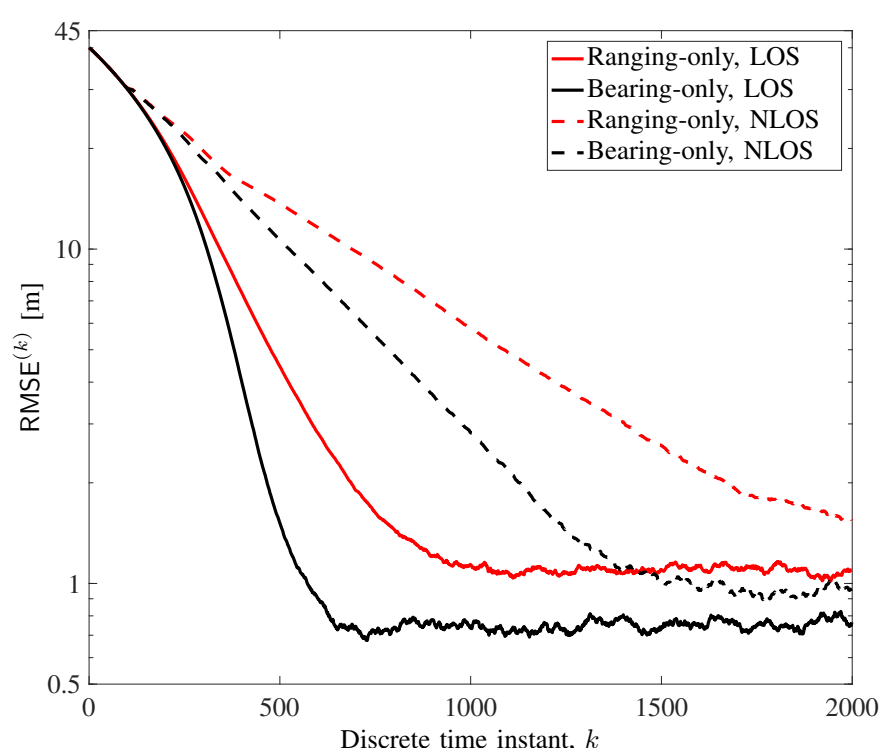

Fig. 7: RMSE as a function of time.

between the estimated and the desired final UAV positions. Navigation constraints are also included in the simulation, with $d_{\mathrm{S}}=0.5 \mathrm{~m}, d_{\mathrm{O}}=5 \mathrm{~m}$, and $v=1 \mathrm{~m} / \mathrm{s}$.

The simulated scenarios are reported in Fig. 6 for a circular shape formation in LOS and NLOS environments and for ranging and bearing measurements. In the plot, the user is indicated with a red triangle, the UAV initial and final positions with white and cyan squares, respectively, and the UAV trajectories with dashed lines. Also some intermediate UAV positions along the trajectories are displayed every 100 time slots with the aim of clarifying that the superimposed trajectories do not coincide with collision events between the UAVs. The obstacles are depicted as grey rectangles. The performance is investigated in terms of RMSE averaged over the number of UAVs, i.e.,

$$
\operatorname{RMSE}^{(k)}=\frac{1}{N} \sum_{i=1}^{N} \sqrt{\frac{1}{N_{\mathrm{MC}}} \sum_{m=1}^{N_{\mathrm{MC}}}\left\|\boldsymbol{\theta}_{i m}^{(k)}-\boldsymbol{\theta}_{i}^{*}\right\|^{2}},
$$

with $N_{\mathrm{MC}}$ being the number of Monte Carlo trials, where at each iteration a different UAV position estimate is generated. In Fig. 7, the red and black curves are for ranging-only and bearing-only measurements, respectively. The dashed lines refer to the presence of obstacles in the simulated environment. The number of UAVs is fixed to $N=9$, the number of Monte Carlo trials to $N_{\mathrm{MC}}=100$, and the multipath bias to $\sigma_{\mathrm{b}}=3 \mathrm{~dB}$. We notice that at the last time instant, the UAVs attain the final desired formation with an average error of less than $2 \mathrm{~m}$. As expected, the NLOS case decreases the performance, especially for the bearing case. A comparison of results based on ranging and bearing measurements is not meaningful because the performance strongly depends on the accuracy of the sensors that acquire the measurements.
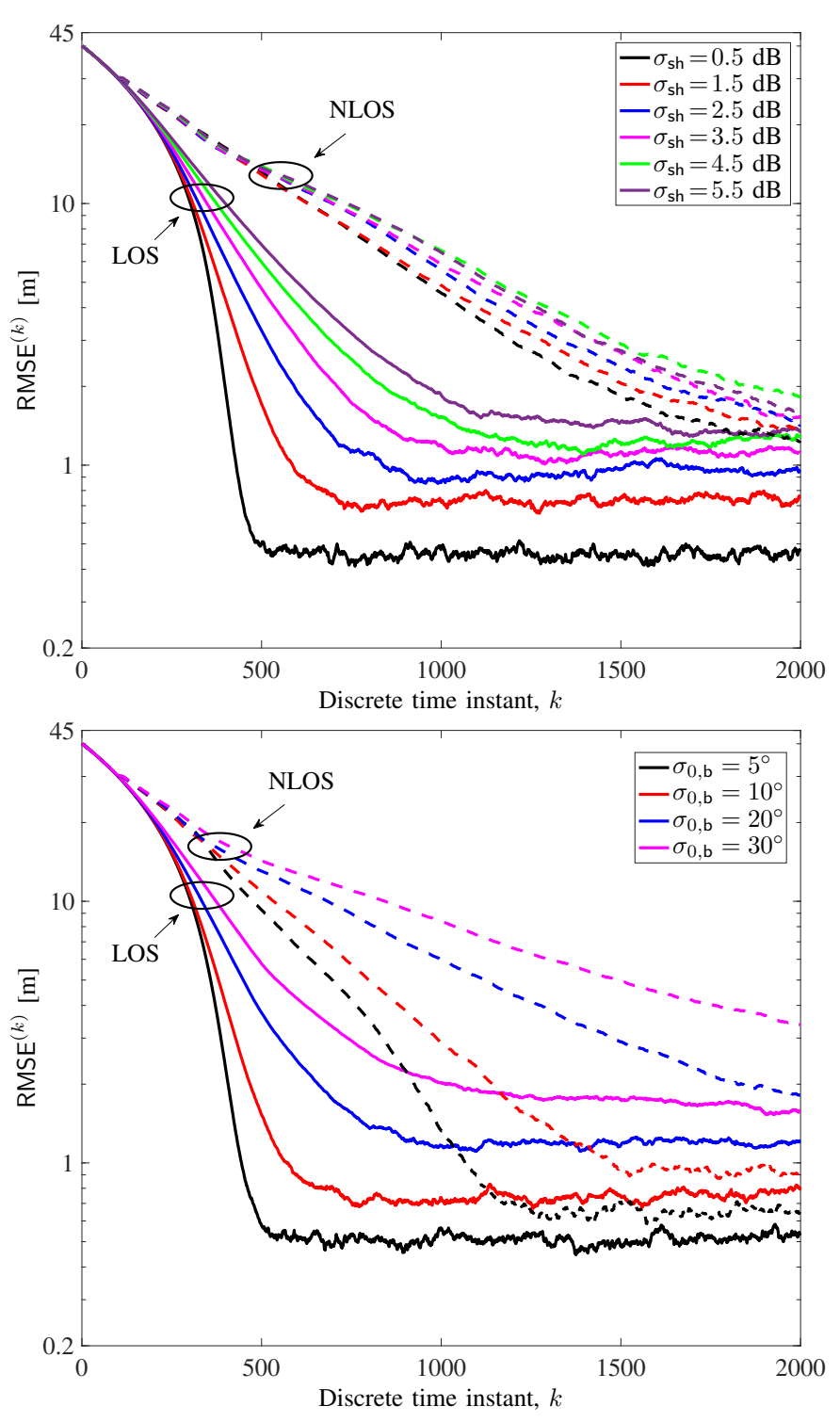

Fig. 8: RMSE as a function of the adopted sensor accuracy. Top: ranging-only measurements; Bottom: bearingonly measurements.

In order to gain insights about the dependence of the performance of the proposed method on this accuracy, we investigated the RMSE of the UAV positions as a function of the sensor accuracy. More specifically, in Fig. 8, we provide the RMSE performance for different choices of the parameters, i.e., $\sigma_{\text {sh }}$, (we suppose that the path loss coefficient is always equal to 2) and $\sigma_{0, \mathrm{~b}}$. As expected, from the results of the ranging case, we observe that the RSSbased distance estimation and, consequently, the positioning become more accurate when the standard deviation of the shadowing decreases. For the bearing case, $\sigma_{0, \mathrm{~b}}=5^{\circ}$ is an accuracy achievable with the adoption of antenna arrays whose integration in drones is today difficult due to size and weight 
constraints. Also in this case, relying on RSS measurements can be a solution at the expense of a degradation in localization and formation performance.

\section{CONCLUSIONS}

In this paper, we investigated the performance of a usercentric network of UAVs in formation and infrastructureless localization in indoor environments. The UAVs are able to exchange measurements with all the other UAVs of the network and communicate the collected observations to a central node, i.e., the user. From ranging and bearing observation models, the user is able to localize the UAVs and to send them navigation commands with the objective of forming a desired final topology. The results of formation accuracy are promising and pave the way to indoor applications using UAVs. In future work, we will consider scenarios with dynamic users.

\section{ACKNOWLEDGMENT}

This work has received funding from the European Union's Horizon 2020 research and innovation programme under the Marie Sklodowska-Curie project AirSens (grant no. 793581) and the support of the NSF under Award CCF-1618999.

\section{REFERENCES}

[1] Y. Liu and R. Bucknall, "A survey of formation control and motion planning of multiple unmanned vehicles," Robotica, pp. 1-29, 2018.

[2] K.-K. Oh, M.-C. Park, and H.-S. Ahn, "A survey of multi-agent formation control," Automatica, vol. 53, pp. 424-440, 2015.

[3] J. M. Sullivan, "Evolution or revolution? The rise of UAVs," IEEE Technol. Soc. Mag., vol. 25, no. 3, pp. 43-49, 2006.

[4] M. Egerstedt and X. Hu, "Formation constrained multi-agent control," IEEE Trans. Robot. Automat., vol. 17, no. 6, pp. 947-951, 2001.

[5] P. Stone and M. Veloso, "Multiagent systems: A survey from a machine learning perspective," Autonomous Robot., vol. 8, no. 3, pp. 345-383, 2000.

[6] T. M. Roehr, F. Cordes, and F. Kirchner, "Reconfigurable integrated multirobot exploration system (RIMRES): Heterogeneous modular reconfigurable robots for space exploration," J. Field Robot., vol. 31, no. 1, pp. 3-34, 2014.

[7] R. Sharma and D. Ghose, "Collision avoidance between UAV clusters using swarm intelligence techniques," Int. J. Syst. Sci., vol. 40, no. 5, pp. 521-538, 2009.

[8] S. Balakirsky et al., "Towards heterogeneous robot teams for disaster mitigation: Results and performance metrics from robocup rescue," $J$. Field Robot., vol. 24, no. 11-12, pp. 943-967, 2007.

[9] Y. Liu and G. Multirobot cooperative learning for semiautonomous control in urban search and rescue applications," J. Field Robot., vol. 33, no. 4, pp. 512-536, 2016

[10] H. Bayerlein, P. De Kerret, and D. Gesbert, "Trajectory optimization for autonomous flying base station via reinforcement learning," in Proc. IEEE 19th Int. Workshop Signal Process. Adv. Wireless Commun. (SPAWC). IEEE, 2018, pp. 1-5.

[11] A. Guerra et al., "Collaborative target-localization and informationbased control in networks of UAVs," in Proc. IEEE 19th Int. Signal Process. Adv. Wireless Commun. (SPAWC). IEEE, 2018, pp. 1-5.

[12] N. Patwari et al., "Locating the nodes: cooperative localization in wireless sensor networks," IEEE Signal Process. Mag., vol. 22, no. 4, pp. 54-69, 2005.

[13] H. Wymeersch, J. Lien, and M. Z. Win, "Cooperative localization in wireless networks," Proc. IEEE, vol. 97, no. 2, pp. 427-450, 2009.

[14] D. B. Jourdan, D. Dardari, and M. Z. Win, "Position error bound for UWB localization in dense cluttered environments," IEEE Trans. Aerosp. Electron. Syst., vol. 44, no. 2, 2008.

[15] S. Gezici, "A survey on wireless position estimation," Wireless personal communications, vol. 44, no. 3, pp. 263-282, 2008.
[16] M. Petitjean, S. Mezhoud, and F. Quitin, "Fast localization of groundbased mobile terminals with a transceiver-equipped UAV," in Proc. 29th Annual Int. Symp. Personal, Indoor and Mobile Radio Commun. (PIMRC). IEEE, 2018.

[17] J. T. Isaacs et al., "Quadrotor control for RF source localization and tracking," in Proc. Int. Conf. Unmanned Aircraft Sys. (ICUAS). IEEE, 2014, pp. 244-252.

[18] —, "GPS-optimal micro air vehicle navigation in degraded environments," in American Control Conf. (ACC), 2014. IEEE, 2014, pp. 1864-1871.

[19] N. B. Priyantha et al., "Anchor-free distributed localization in sensor networks," in Proc. Int. Conf. Embedded Netw. Sensor Sys. ACM, 2003, pp. 340-341.

[20] R. L. Moses, D. Krishnamurthy, and R. M. Patterson, "A selflocalization method for wireless sensor networks," J. Adv. Signal Process. (EURASIP), vol. 2003, no. 4, p. 839843, 2003.

[21] H. L. Van Trees, Detection, Estimation, and Modulation Theory. John Wiley \& Sons, 2004.

[22] D. G. Luenberger, Y. Ye et al., Linear and Nonlinear Programming. Springer, 1984, vol. 2. 